\title{
Regeneration of the MPTP-Lesioned Dopaminergic System after Convection-Enhanced Delivery of AAV2-GDNF
}

\author{
Adrian P. Kells, ${ }^{1}$ Jamie Eberling, ${ }^{2}$ Xiaomin Su, ${ }^{1}$ Philip Pivirotto, ${ }^{1}$ John Bringas, ${ }^{1}$ Piotr Hadaczek,,${ }^{1}$ Wade C. Narrow, ${ }^{3}$ \\ William J. Bowers, ${ }^{3}$ Howard J. Federoff, ${ }^{4}$ John Forsayeth, ${ }^{1}$ and Krystof S. Bankiewicz ${ }^{1}$ \\ ${ }^{1}$ Department of Neurological Surgery, University of California San Francisco, San Francisco, California 94103, ${ }^{2}$ Department of Molecular Imaging and \\ Neuroscience, Lawrence Berkeley National Laboratory, Berkeley, California 94720, ${ }^{3}$ Department of Neurology, Center for Neural Development and Disease, \\ University of Rochester Medical Center, Rochester, New York 14642, and ${ }^{4}$ Departments of Neurology and Neuroscience, Georgetown University Medical \\ Center, Washington, DC 20007
}

Clinical studies to date have failed to establish therapeutic benefit of glial cell-derived neurotrophic factor (GDNF) in Parkinson's disease (PD). In contrast to previous nonclinical neuroprotective reports, this study shows clinically relevant and long-lasting regeneration of the dopaminergic system in rhesus macaques lesioned with 1-methy-4-phenyl-1,2,3,6-tetrahydropyridine 3-6 months before GDNF gene delivery (AAV2-GDNF). The observed progressive amelioration of functional deficits, recovery of dopamine, and regrowth of fibers to the striatal neuropil demonstrate that high GDNF expression in the putamen promotes restoration of the dopaminergic system in a primate model of advanced PD. Extensive distribution of GDNF within the putamen and transport to the severely lesioned substantia nigra, after convection-enhanced delivery of AAV2-GDNF into the putamen, indicates anterograde transport via striatonigral connections and is anticipated to occur in PD patients. Overall, these data demonstrate nonclinical neurorestoration after putaminal infusion of AAV2-GDNF and suggest that clinical investigation in PD patients is warranted.

\section{Introduction}

Because almost all Parkinson's disease (PD) patients are clinically diagnosed only after substantial degeneration of the dopaminergic system, there is a vital need to develop neurorestorative treatments. Current PD therapies provide only timelimited symptomatic relief of parkinsonian motor symptoms and do not prevent disease progression (Chen and Swope, 2007; Davie, 2008). Neurotrophic factor therapy has long been proposed as a way to mitigate neurodegenerative disease processes and potentially stimulate regeneration of degenerated neurons (Dawbarn and Allen, 2003). Glial-derived neurotrophic factor (GDNF) is involved in prenatal development of mesencephalic dopaminergic neurons (Lin et al., 1993; Granholm et al., 2000) and is critical for postnatal survival of these neurons (Pascual et al., 2008). Therefore, GDNF and its homolog, Neurturin, have received considerable attention as therapeutic agents for the treatment of PD (Peterson and Nutt, 2008). Unfortunately, despite promising data generated in neurotoxicant models in rodents and nonhuman primates (NHP), clinical studies in PD patients so far have failed to meet primary efficacy endpoints. This suggests a deficiency either in the predictive ability of previously used nonclinical models or in the ability to translate research protocols into effective clinical procedures.

\footnotetext{
Received Feb. 19, 2010; revised April 15, 2010; accepted June 2, 2010.

This study was funded by a National Institutes of Health-National Institute of Neurological Disorders and Stroke Cooperative Research Agreement U54 NS045309.

Correspondence should be addressed to Krystof S. Bankiewicz, Department of Neurological Surgery, University of California San Francisco, 1855 Folsom Street, San Francisco, CA 94103. E-mail: krystof.bankiewicz@ucsf.edu. D0I:10.1523/JNEUROSCI.0942-10.2010

Copyright $\odot 2010$ the authors $\quad 0270-6474 / 10 / 309567-11 \$ 15.00 / 0$
}

The failure of previous attempts to efficaciously deliver neurotrophic factors to the human putamen of PD patients has been attributed to ineffective delivery (Patel and Gill, 2007). Clinical studies have revealed the significant technical challenges associated with intracranial delivery to targeted regions. Although less of an issue in small animal models, efficient distribution at physiologically relevant levels within the substantially larger adult human brain is critical for the efficacy of any neurotrophic factor therapy (Yin et al., 2009a). To facilitate translation from NHP to clinical studies, our group has invested considerable effort in the development of reflux-resistant cannulae and infusion protocols that enable delivery of clinically relevant volumes to precisely targeted structures within the brain (Varenika et al., 2009). The recent development of convection-enhanced delivery (CED) techniques, including clinically compatible real-time MRI-based targeting and visualization of vector infusions, will assist in translating this current study into an anticipated phase I AAV2-GDNF study for mid-stage PD (Fiandaca et al., 2008).

The most universally accepted NHP model of PD pathology involves 1-methy-4-phenyl-1,2,3,6-tetrahydropyridine (MPTP)induced lesioning of the dopaminergic system (Bankiewicz et al., $1986,2001)$. We have previously demonstrated that the neurodegenerative process in this model occurs over a 4-week period after acute MPTP dosing (Eberling et al., 1997). Consequently, any therapeutic intervention before (Eslamboli et al., 2003, 2005; Li et al., 2003) or during (Kordower et al., 2000, 2006; Oiwa et al., 2006) this extended period of neurotoxin-induced lesioning should be viewed in the context of neuroprotection rather than restoration. This distinction is essential for future clinical study with PD patients who already have extensive nigrostriatal degen- 
eration and will likely require a neurorestorative treatment to gain meaningful clinical improvement. To our knowledge, the present study is unique in that initiation of neurotrophic factor gene delivery was delayed until lesioning of the nigrostriatal pathway was complete to ensure a direct assessment of neuronal repair that is consistent with the expectation of the ongoing or future clinical trials testing neurorestorative potential of GDNF family of growth factors in PD.

\section{Materials and Methods}

Experimental plan. This study was designed to assess the safety and efficacy of AAV2-GDNF in a cohort of adult rhesus macaques rendered hemi-parkinsonian by MPTP administration. The animals were assessed in-life for up to 24 months after AAV2-GDNF or sham delivery. Three macaques were killed for early toxicity and distribution analysis at 1 month and the remaining animals were killed in pairs at $6,14,20$, or 24 months.

Experimental subjects. The protocol was approved by the Institutional Animal Care and Use Committee at the University of California San Francisco and the Animal Welfare and Research Committee at the Lawrence Berkeley National Laboratory. Fourteen male rhesus monkeys $(8-14 \mathrm{~kg})$, and one female rhesus monkey $(7 \mathrm{~kg})$ were lesioned with MPTP as previously described (Bankiewicz et al., 1986, 2000; Eberling et al., 1998). Briefly, MPTP lesioning consisted of one or two right intracarotid artery infusions of $2.0-4.0 \mathrm{mg}$ of MPTP- $\mathrm{HCl}$, followed by additional intravenous administrations of $0.2-0.5 \mathrm{mg} / \mathrm{kg}$ doses of $\mathrm{MPTP}-\mathrm{HCl}$. This method of lesioning produces nearly a complete dopaminergic lesion on the side of carotid artery infusion (right hemisphere) and a partial lesion in the contralateral (left) hemisphere of the brain, creating a humane and stable model of PD able to self-maintain within animals. In addition, this hemi-parkinsonian model allows investigation of a severe dopaminergic lesion reminiscent of advanced PD on the ipsilateral hemisphere, whereas the contralateral partially lesioned hemisphere models earlier stages of PD. Intravenous dosing with MPTP continued until each animal showed bilateral parkinsonian signs and a clinical rating scale (CRS) score between 21 and 26, as described below in Clinical rating scale. Only macaques with CRS scores that were stable for at least 3 months before treatment were enrolled in the study. Macaques were randomly assigned to receive bilateral administration of either AAV2-GDNF $\left(9.9 \times 10^{11}\right.$ vector genomes; $\left.n=8\right)$ or PBS $(n=7)$ into the putamen via CED that involved the ramped infusion of $75 \mu$ l into two stereotactic sites in each putamen (precommissural and postcommissural). In previous studies, we have demonstrated that AAV2 vectors encoding control genes (i.e., LacZ) had no therapeutic effect on MPTPlesioned macaques (Bankiewicz et al., 2006; Forsayeth et al., 2006). To enable complete assessment of both the AAV2 vector and GDNF gene product, the control macaques in this study received only a sham PBS infusion.

AAV2-GDNF vector. The AAV2-GDNF vector construct contained cDNA for human GDNF under the control of a cytomegalovirus promoter with a $3^{\prime}$ human growth hormone polyadenylation sequence. The expression cassette was flanked by AAV2 inverted terminal repeats and packaged into recombinant AAV2 vector particles (Avigen) by means of a standard helper free transfection method, as previously described (Matsushita et al., 1998; Wright et al., 2003).

Clinical rating scale. All ratings were performed by a single investigator blinded to the experimental conditions. The modified Parkinson's CRS used here was developed in our laboratory and closely approximates those reported in the literature (Imbert et al., 2000). The scale evaluates 14 parkinsonian features, each of which receives a score from 0 to 3 in order of increasing severity $(0=$ normal, $1=$ mild, $2=$ moderate, $3=$ severe). Individual scores are summed to arrive at a final score. Features evaluated include tremor (right and left sides), locomotion, "freezing," fine motor skills (right and left hand), bradykinesia (right and left sides), hypokinesia, balance, posture, startle response, and gross motor skills (right and left hand). Normal animals score in the range $0-4$, and severely parkinsonian monkeys score $>20$. CRS assessments were performed after MPTP lesioning to determine a stable baseline before treatment. Animals were assessed both with and without L-dopa administration. Individual CRS ratings over 5-10 separate days were averaged for each time point to provide unbiased CRS scores. To assess the L-dopa response, the animals were treated with a twice-daily intramuscular injection of L-dopa at a therapeutic level determined pretreatment and maintained throughout the study $(3-10 \mathrm{mg} / \mathrm{kg}$ ). CRS ratings were made 30 min after L-dopa administration.

Positron emission tomography. Dopaminergic function was measured by $6-\left[{ }^{18} \mathrm{~F}\right]$ fluoro-L- $m$-tyrosine-positron emission tomography (FMTPET) as previously described (Eberling et al., 2009). FMT-PET was performed 3 months before (baseline) and 6 months after treatment for all animals. The long-term survival animals treated with AAV2-GDNF were rescanned 23 months after vector infusion. Parametric images of FMT influx $\left(K_{\mathrm{i}}\right)$ from both time points were generated and coregistered to identify functional changes after treatment. FMT influx in the putamen was thereby measured before and after treatment for each monkey.

Necropsy. Three macaques (two AAV2-GDNF-treated and one PBS control) were killed 1-month after AAV2-GDNF or PBS delivery. Pairs of the remaining animals from each group were killed after treatment at the following time intervals: 6, 14, and 24 months (AAV2-GDNF) and 6, 14, and 20 months (PBS). In brief, the unfixed brain was removed from the animal after perfusion with PBS only and sectioned into $3 \mathrm{~mm}$ coronal blocks. One putamen block and one block containing the substantia nigra were fresh-frozen for biochemical and protein analyses. All other blocks were fixed with $4 \%$ paraformaldehyde and processed for immunohistochemical staining.

High-performance liquid chromatography analysis. Fresh tissue punches from the putamen were homogenized by sonication in $250 \mu \mathrm{l}$ of $0.4 \mathrm{M}$ perchloric acid and then centrifuged for $15 \mathrm{~min}$ at 13,000 rpm. The supernatant solutions were filtered and the concentration of dopamine, homovanillic acid (HVA), and 3,4-dihydroxyphenylacetic acid (DOPAC) were determined by high-performance liquid chromatography (HPLC) as previously described (Johnston et al., 2009). Total protein was determined in the pellet fractions after resuspension in $0.5 \mathrm{M} \mathrm{NaOH}$ via a DC protein assay kit (Bio-Rad).

GDNF ELISA. The tissue concentration of GDNF was determined from putamen and substantia nigra tissue punches with a commercially available GDNF ELISA kit (Promega). Fresh-frozen tissue punches were homogenized in $250 \mu$ l of lysis buffer (Tropix) supplemented with protease inhibitors (Complete mini; Roche Molecular Biochemicals). ELISA was performed as per the manufacturer's guidelines except for color development and absorbance reading, which was replaced with a more sensitive chemiluminescent substrate kit (SuperSignal ELISA pico chemiluminescent substrate; Thermo Scientific). Total protein concentration was determined with a DC protein assay kit (Bio-Rad).

Immunohistochemical staining. Coronal brain blocks fixed in $4 \%$ paraformaldehyde were either sectioned to $40 \mu \mathrm{m}$ on a sliding microtome or paraffin-embedded and sectioned to $5 \mu \mathrm{m}$. Immunohistochemical staining was performed on sections from the caudate-putamen through to the mid-brain region with primary antibodies for GDNF (R\&D Systems), tyrosine hydroxylase (TH; Millipore Bioscience Research Reagents), GFAP (Millipore Bioscience Research Reagents), and Iba1 (Biocare Medical). Polymer-based detection kits (Biocare Medical) and diaminobenzidine (DAB; Vector Laboratories) were used to complete the staining.

Tyrosine hydroxylase analysis. The extent of dopaminergic innervation of the putamen was assessed by densitometry analysis of $5 \mu \mathrm{m}$ paraffin sections stained for TH. Images were taken of four different regions of each putamen in a single coronal section-dorsomedial, dorsolateral, central, and ventral aspects-with a $20 \times$ objective lens. Three AAV2GDNF-treated macaques and three PBS controls from the 14- and 24month groups were included in this analysis. Images of the corpus callosum and cerebral cortex, devoid of TH staining, were also captured. Image $(\mathrm{NIH})$ software was used to analyze the images by thresholding the images to limit measurements to the TH-positive fibers and then obtaining an integrated density measurement by multiplying the $\mathrm{TH}$ stained area by the mean pixel intensity. To account for any staining variation between sections, the density measurements for the putaminal images were divided by the mean density of the corpus callosum and 

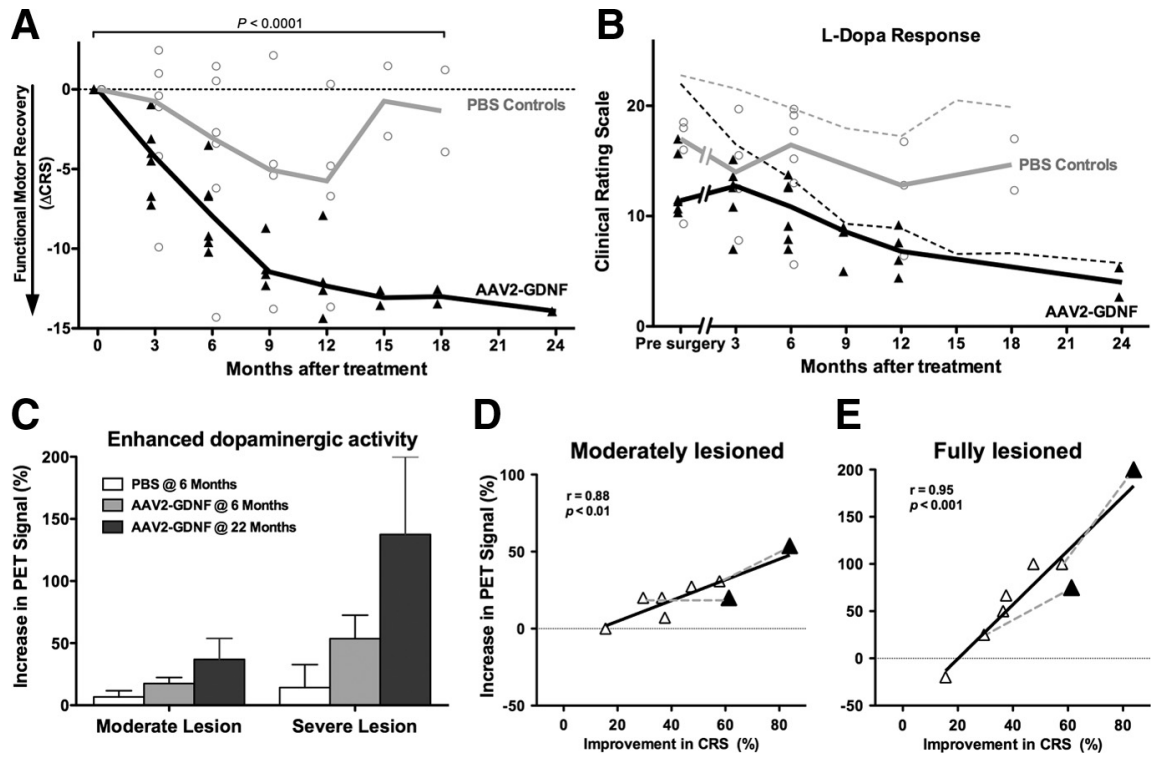

Figure 1. Effect of AAV2-GDNF infusion on parkinsonian symptoms and FMT-PET. A, CRS scores in MPTP-lesioned macaques treated with AAV2-GDNF (black triangles) were significantly reduced compared with the PBS controls (gray circles). Two-way ANOVA statistics for the effect of treatment are shown for the three different survival periods. $B$, CRS scores after L-dopa administration (symbols and solid lines) were lower than the OFF L-dopa scores (dashed lines) for both AAV2-GDNF and PBS controls. $\boldsymbol{C}$, Enhanced dopaminergic activity in the putamen after AAV2-GDNF delivery. FMT-PET was considerably increased bilaterally in AAV2-GDNF-treated macaques (light and dark gray bars) compared with the PBS controls. Twenty-two months after AAV2-GDNF delivery, the PET signal was further increased with the severely lesioned putamen showing a much greater percentage increase in FMT uptake than the moderately lesioned putamen. Bars are mean \pm SEM. $\boldsymbol{D}, \boldsymbol{E}$, Direct correlations between the increase in FMT-PET and improvements in CRS were observed for both the moderately lesioned left $(\boldsymbol{D})$ and severely lesioned right $(\boldsymbol{E})$ putamen. These correlations were evident 6 months after treatment (open triangles) and were undiminished at 22 months (closed triangles). Dashed lines connect the 6 - and 22-month measurements for the same macaques. Figure updated and modified from our preliminary report (Eberling et al., 2009).

cerebral cortex from the same section. Microscope, camera, and software settings remained consistent for all images.

\section{Results}

\section{Amelioration of functional motor deficits}

MPTP-induced lesioning of the dopaminergic system in the adult NHP results in impaired motor control comparable to parkinsonian movement deficits and provides a reliable model for investigating restorative approaches for treating PD. The hemi-lesioned model used in this study produces a complete dopaminergic lesion in the right hemisphere and a partial lesion in the left hemisphere. A modified parkinsonian CRS was used to serially measure the extent of motor impairment after the initial intracarotid infusion of MPTP. All animals subsequently received systemic intravenous dosing of MPTP until they displayed bilateral motor impairment and achieved a CRS score between 17 and 26 that remained stable for at least 3 months before treatment.

All AAV2-GDNF-treated macaques displayed considerable improvement in their CRS scores in the period between 6 and 24 months of the study, whereas PBS-treated control macaques (with the exception of one outlier) exhibited significantly less improvement in their CRS scores (Fig. 1A). Although no reason for the substantial improvement of the outlier control animal was evident, it has previously been observed that infrequently an MPTP-lesioned NHP will show spontaneous behavioral recovery (Bankiewicz et al., 2006). Pairs of AAV2-GDNF-treated and PBS control macaques were killed at 6, 14, and 20 (PBS controls) or 24 (AAV2-GDNF) months after treatment to provide a timedependent immunohistochemical assessment of safety and tox- icity. This protocol restricted statistical comparison of the CRS scores, although two-way ANOVA of CRS recovery revealed a very significant effect of treatment over the complete dataset $(p<0.0001)$. When the spontaneously recovered control macaque was excluded, separate two-way repeated measure ANOVA analyses showed significant treatment effects for animals remaining at each endpoint (6 months, $p=0.013 ; 12$ months, $p=0.020 ; 18$ months, $p=0.050)$. Improvements in CRS scores from pretreatment baseline scores ( $\Delta$ CRS) were evident 3 months after treatment $(\triangle \mathrm{CRS}$ AAV2-GDNF, $-4.4 \pm 1.0$; PBS, $-0.5 \pm 1.1 ; t$ test, $p=$ 0.036 ), with progressive CRS improvement observed throughout the 9 month period after AAV2-GDNF treatment with a mean $56 \pm 4 \%$ reduction in CRS score $(\triangle \mathrm{CRS}$ AAV2-GDNF, $-11.0 \pm 0.8$; PBS, $-2.7 \pm 2.4 ; t$ test, $p=0.013)$.

\section{L-Dopa administration}

To address the possibility that AAV2-GDNF treatment might be unsafe if combined with dopamine replacement therapy, we administered therapeutic doses of L-dopa to the macaques at various times throughout the study. All animals manifested measureable clinical responses to L-dopa with an improvement relative to their OFF L-dopa CRS scores (Fig. $1 B$ ). The PBS-infused controls demonstrated a consistent L-dopa response throughout the study, with a mean improvement in CRS score of $18 \%$ at both 6 and 12 months after treatment. This L-dopa response was less than the response presurgery (29\% CRS improvement) and at 3 months (25\%), probably related to the small improvement in OFF L-dopa CRS score exhibited by these controls after surgery. AAV2-GDNF-treated macaques exhibited a similar L-dopa response to the PBS controls at $3(25 \%)$ and 6 months (19\%), but the CRS score was less affected at 12 months (11\%) because of the already considerable CRS improvements due to GDNF activity. Macaques were maintained on a consistent therapeutic dose of L-dopa without any serious adverse effects. However, both AAV2-GDNF- and PBS-treated animals were more likely to become distracted from performing the tasks required to complete the CRS assessment when receiving L-dopa. One of the AAV2-GDNF-treated macaques (NHP437) displayed L-dopa-induced dyskinetic movement in its left leg; however, this was not observed in any of the other macaques and may be related to the specific distribution of GDNF in this one animal (supplemental Fig. S1, available at www.jneurosci.org as supplemental material).

\section{Enhanced dopaminergic activity}

PET scans were performed before and after treatment to assess in-life changes in dopaminergic terminal integrity. As detailed in our earlier report (Eberling et al., 2009), a bilateral increase in FMT uptake was observed in the AAV2-GDNF-treated macaques 6 months after vector infusion where $K_{\mathrm{i}}$ values were increased by $18 \pm 5 \%$ in the moderately lesioned putamen and by $54 \pm 19 \%$ in the severely lesioned putamen. PBS control macaques did not 


\begin{tabular}{|c|c|c|c|c|c|}
\hline & \multicolumn{2}{|c|}{ Left (moderately lesioned) } & \multicolumn{2}{|c|}{ Right (severely lesioned) } & \multirow[b]{2}{*}{ Naïve $^{a}$} \\
\hline & AAV2-GDNF & PBS controls & AAV2-GDNF & PBS controls & \\
\hline \multicolumn{6}{|c|}{ Catecholamine levels (ng/mg total protein) } \\
\hline Dopamine & $204 \pm 33^{* *}$ & $68 \pm 8$ & $1.2 \pm 0.5$ & $0.8 \pm 0.3$ & $240 \pm 19$ \\
\hline HVA & $473 \pm 51^{* * * *}$ & $149 \pm 11$ & $51 \pm 12^{*}$ & $20 \pm 6$ & $270 \pm 27$ \\
\hline DOPAC & $40 \pm 5^{* * *}$ & $11 \pm 1$ & $0.7 \pm 0.2$ & $0.3 \pm 0.1$ & $\mathrm{n} / \mathrm{a}$ \\
\hline \multicolumn{6}{|c|}{ Rate of dopamine turnover (metabolite:dopamine ratio) } \\
\hline HVA:DA & $2.6 \pm 0.4$ & $2.3 \pm 0.2$ & $52 \pm 6^{* *}$ & $30 \pm 2$ & 1.1 \\
\hline DOPAC:DA & $0.21 \pm 0.03$ & $0.18 \pm 0.03$ & $0.66 \pm 0.06^{* *}$ & $0.41 \pm 0.05$ & $\mathrm{n} / \mathrm{a}$ \\
\hline
\end{tabular}

DA, Dopamine; $n / a$; not applicable. Data are mean \pm SEM. Two-tailed unpaired $t$ test compared to PBS controls: ${ }^{*} p<0.05 ;{ }^{* *} p<0.01 ;{ }^{* * *} p<0.001 ;{ }^{* * * *} p<0.0001$.

${ }^{a}$ Prior analysis of naïve adult rhesus macaques by Oiwa et al. (2003).

show a significant change from baseline in FMT uptake in either hemisphere. The increased FMT uptake in AAV2-GDNF-treated macaques was directly correlated with improvements in CRS scores at 6 months. Additional PET scans performed 22 months after AAV2-GDNF treatment in the two long-term survival macaques showed further increases in FMT uptake compared with the baseline PET (left putamen $37 \pm 17 \%$ increase; right putamen $138 \pm 63 \%$ increase) (Fig. 1C). A larger increase in FMT uptake between 6 and 22 months was observed in the severely lesioned hemisphere $(40-50 \%$ increase) than in the moderately lesioned hemisphere $(0-18 \%$ increase). The direct correlations between the increase in PET signal and improvement in CRS were maintained at 22 months for both the left (Pearson's $r=0.88$; $p<0.01$ ) and right (Pearson's $r=0.95, p<0.001$ ) hemispheres (Fig. $1 D, E$ ).

To directly assess changes in dopamine metabolism after AAV2-GDNF treatment, putaminal levels of dopamine and dopamine metabolites (HVA and DOPAC) were quantified (Table 1). Fresh tissue punches were collected postmortem and analyzed by HPLC. We found no time-dependent changes in dopamine (Fig. $2 A, B$ ), HVA, or DOPAC levels within the AAV2-GDNF and PBS treatment groups killed at different time intervals (6-24 months) after treatment. As expected, after MPTP lesioning (Oiwa et al., 2003), control macaques showed reduced dopamine levels in both hemispheres with almost complete loss of dopamine in the severely lesioned right putamen $(0.8 \pm 0.3 \mathrm{ng} / \mathrm{mg}$ protein) and an $\sim 70 \%$ loss in the moderately lesioned left putamen ( $68 \pm 8 \mathrm{ng} / \mathrm{mg}$ protein) relative to dopamine levels in naive macaques (Oiwa et al., 2003). Delivery of AAV2-GDNF into the putamen induced a threefold increase in dopamine within the moderately lesioned left putamen $(204 \pm 33 \mathrm{ng} / \mathrm{mg}$ protein; unpaired $t$ test, $p<0.01$ ), almost completely restoring dopamine to nonlesioned levels ( $240 \pm 19 \mathrm{ng} / \mathrm{mg}$ protein) (Oiwa et al., 2003). In contrast, dopamine levels in the severely lesioned right putamen were not substantially changed by AAV2-GDNF treatment $(1.2 \pm 0.5 \mathrm{ng} / \mathrm{mg}$ protein; unpaired $t$ test, $p=0.4)$. Levels of dopamine metabolites were, however, substantially increased in both the severe and moderately lesioned hemispheres (Table 1). By calculating the ratio of dopamine metabolites (HVA and DOPAC) to dopamine, a relative rate of dopamine turnover can be determined. Naive macaques typically have an HVA:dopamine ratio close to $1: 1$ and this ratio is substantially increased after MPTP lesioning. Consequently, both the AAV2-GDNF- and PBS-treated groups displayed bilaterally increased rates of dopamine turnover that were not significantly different between the three time points (Table 1; Fig. 2C,D). Nevertheless, AAV2GDNF delivery resulted in a further enhancement (unpaired $t$ test, $p<0.01)$ of both the HVA:dopamine and DOPAC:dopamine ratios relative to the PBS controls within the severely lesioned putamen (Table 1). AAV2-GDNF treatment did not significantly alter the rate of dopamine turnover in the partially lesioned putamen, with equivalent increases of dopamine, HVA, and DOPAC.

To further analyze the effect of AAV2-GDNF on dopamine in the putamen, we compared the level of dopamine and HVA with the corresponding rate of dopamine turnover in each hemisphere. In the severely lesioned hemisphere, there was a strong linear correlation between dopamine and HVA (Pearson's $p<$ 0.0001) (Fig. 2E), with AAV2-GDNF treatment largely resulting in an elevation of HVA levels but only a slight change in the actual amount of dopamine. This is reflected in the significantly enhanced rate of dopamine turnover (HVA:dopamine ratio) after AAV2-GDNF treatment that was highest in the AAV2-GDNFtreated macaques with the lowest dopamine levels (Fig. 2G). In contrast, significantly increased HVA levels in the moderately lesioned hemisphere after AAV2-GDNF treatment were generally matched by increased dopamine levels (Fig. $2 F$ ) with the result that the HVA:dopamine ratio for the majority of animals was equivalent to the PBS-treated controls (Fig. $2 \mathrm{H}$ ). Overall, this resulted in a strong correlation between dopamine levels and the HVA:dopamine ratio, where the turnover of dopamine was reduced to a relatively normal rate $(\sim 2: 1)$ as the amount of dopamine increased to levels found in naive macaques.

\section{GDNF expression}

We investigated the extent of GDNF distribution by performing immunohistochemical staining on free-floating coronal sections from the caudate-putamen and mid-brain regions of the AAV2GDNF-treated macaques (Fig. 3). Extensive GDNF expression was present at the sites of CED infusion and covered a large proportion of the surrounding putamen in 12 of 16 infused hemispheres (Table 2). Without real-time imaging to monitor vector delivery (Fiandaca et al., 2009; Su et al., 2010), it was not evident why GDNF expression was limited in the other three cases, although actual positioning of cannulae within the putamen largely determines the extent of vector distribution and, hence, vector transduction within the putamen (Yin et al., 2009b). Consistent with previous delivery of AAV2-GDNF vectors into the putamen, very few GDNF-positive cells in the putamen were identifiable and most of the GDNF immunostaining did not colocalize with any cellular structures (supplemental Fig. S2 A, available at www. jneurosci.org as supplemental material). However, in our extensive experience with AAV2 vectors encoding other transgenes, including GFP (green fluorescent protein) and AADC (aromatic L-amino acid decarboxylase) (Bankiewicz et al., 2000; Hadaczek et al., 2006; Cunningham et al., 2008), we have demonstrated extensive transduction of striatal neurons that matched the pattern of GDNF distribution observed in the present animals. GDNF-positive neurons were identified in globus pallidus, subthalamic nucleus, and substantia nigra, consistent with axonal 

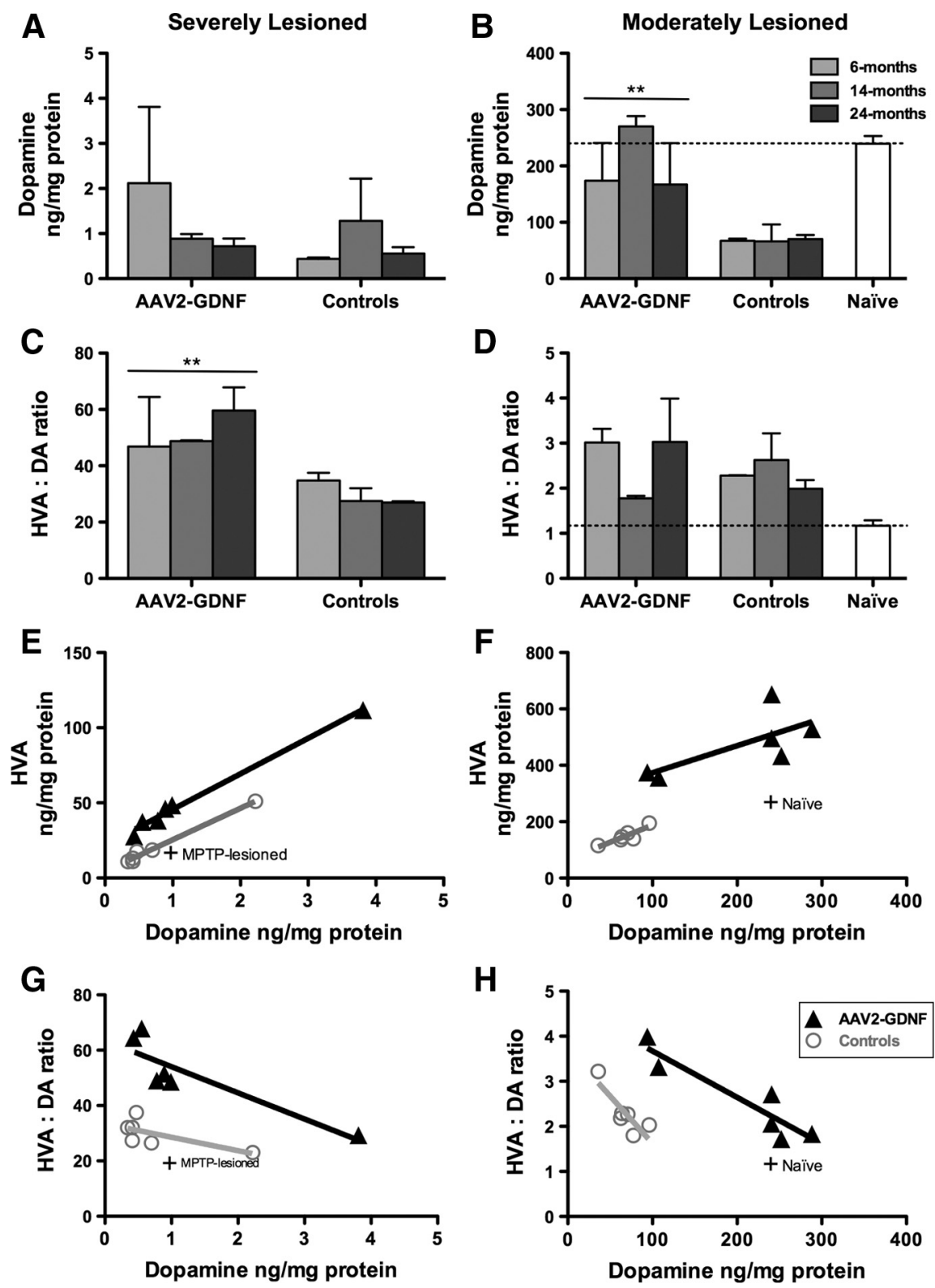

Figure 2. Dopamine and metabolites in severely and moderately lesioned putamen. $A-D, H P L C$ assay of dopamine (DA) levels $(A, B)$ and HVA:dopamine ratios $(\boldsymbol{C}, \boldsymbol{D})$ in the putamen of AAV2-GDNF and PBS control-treated macaques at 6-, 14-, and 24-month endpoints. $\boldsymbol{E}-\boldsymbol{H}$, Correlation analysis of HVA versus dopamine $(\boldsymbol{E}, \boldsymbol{F})$ and the rate of dopamine turnover (HVA:dopamine ratio) versus dopamine $(\boldsymbol{G}, \boldsymbol{H})$. Previously reported data (Oiwa et al., 2003) from naive and parkinsonian macaques are included for comparison [white bars in $\boldsymbol{B}$ and $\boldsymbol{D}$, labeled points (+) in $\boldsymbol{E}-\boldsymbol{H}$ ]. ${ }^{* *} p<0.01$, two-tailed unpaired $t$ test compared to PBS controls.

transport of the AAV2-GDNF particles and/or GDNF protein (Fig. 3 and supplemental Fig. S2 B-D, available at www.jneurosci. org as supplemental material). Substantial bilateral transport of GDNF to the substantia nigra was observed at all time points, indicating that transport to the mid-brain is not dependent on the integrity of the dopaminergic nigrostriatal axonal projections, which were severely degenerated in the right hemisphere.

Quantification of GDNF protein in the putamen and substantia nigra was obtained by ELISA of fresh tissue punches. GDNF levels in the putamen as high as $130 \mathrm{ng} / \mathrm{mg}$ total protein (mean, $24 \pm 10 \mathrm{ng} / \mathrm{mg}$ total protein) were detected. No difference in GDNF expression was found between hemispheres. GDNF levels in the substantia nigra were similar for each hemisphere [left (moderately lesioned), $1.7 \pm 0.8 \mathrm{ng} / \mathrm{mg}$ protein; right (severely lesioned) $1.5 \pm 0.4 \mathrm{ng} / \mathrm{mg}$ protein]. No GDNF protein expression was detected in any of the PBS-treated controls.

Restoration of tyrosine hydroxylasepositive fibers and neurons

To investigate anatomical changes in the nigrostriatal pathway after AAV2-GDNF treatment, we performed immunohistochemical staining for TH (Figs. 3B, 4). The severity of the MPTP-lesion was evident in the right caudate and putamen with almost complete loss of TH-positive fibers in the PBS controls. In contrast, AAV2GDNF-treated macaques showed extensive $\mathrm{TH}$-positive fiber networks within the lesioned putamen, partially along the ventral and medial regions. Increased $\mathrm{TH}$ positive fiber staining was observed in the left, moderately lesioned hemisphere of AAV2-GDNF-treated macaques, specifically in the putamen but also extending into the white matter tracts surrounding the globus pallidus. Integrated areadensity measurements were used to quantify the enhanced TH-staining within the putamen (Fig. 5). A significant increase in relative $\mathrm{TH}$-staining was found in both moderately lesioned (staining intensity: AAV2-GDNF, $520 \pm 50$; PBS, $190 \pm 20$; unpaired $t$ test, $p<0.01$ ) and severely lesioned (AAV2-GDNF, $50 \pm 10$; PBS, $5.5 \pm 0.2 ; p<0.05)$ putamen of AAV2GDNF-treated macaques compared with the PBS controls. Enhanced TH staining was observed across all regions of the moderately lesioned left hemisphere (twofold to threefold increase compared with PBS controls). However, in the severely lesioned right hemisphere, the ventral region of the putamen showed the greatest enhancement ( $\sim 30$-fold increase), with the medial and central areas of the putamen also displaying a significant sixfold increase in TH staining.

In addition to the overall increase in TH-staining intensity in the caudate-putamen, we found large, TH-positive structures primarily within the moderately lesioned hemisphere of AAV2-GDNF-treated animals at all time points (supplemental Fig. S3, available at www.jneurosci.org as supplemental material). We did not observe any significant change in the number, size, or distribution of these structures between the 6-, 14-, and 24-month animals. These TH-positive profiles were considerably less abundant in the severely lesioned hemisphere and were not observed after AAV2-GDNF delivery to intact aged macaques (Su et al., 2009). Further investigation of these TH-positive profiles revealed that they lacked nuclei (DAPI staining), did not colocalize with ubiquitin, and were not associated with activated microglia. Therefore, in agreement with our earlier assessment, we believe these profiles are caused by GDNFinduced enhancement of partially degenerated dopaminergic fibers. A potentially analogous enlargement of $\mathrm{TH}$-positive fibers 


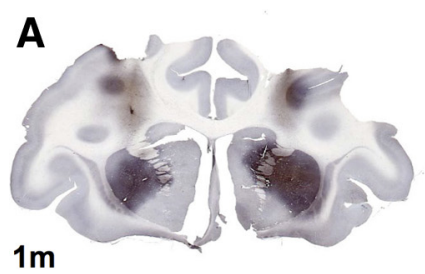

B

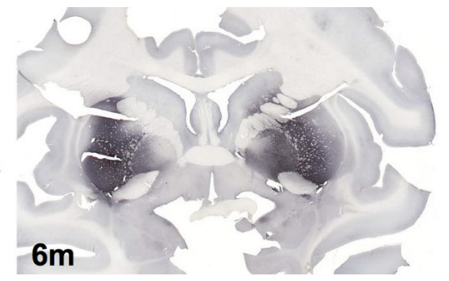

AAV2-GDNF
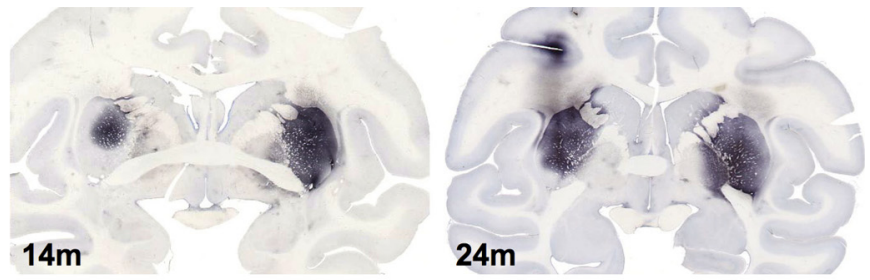

PBS-control
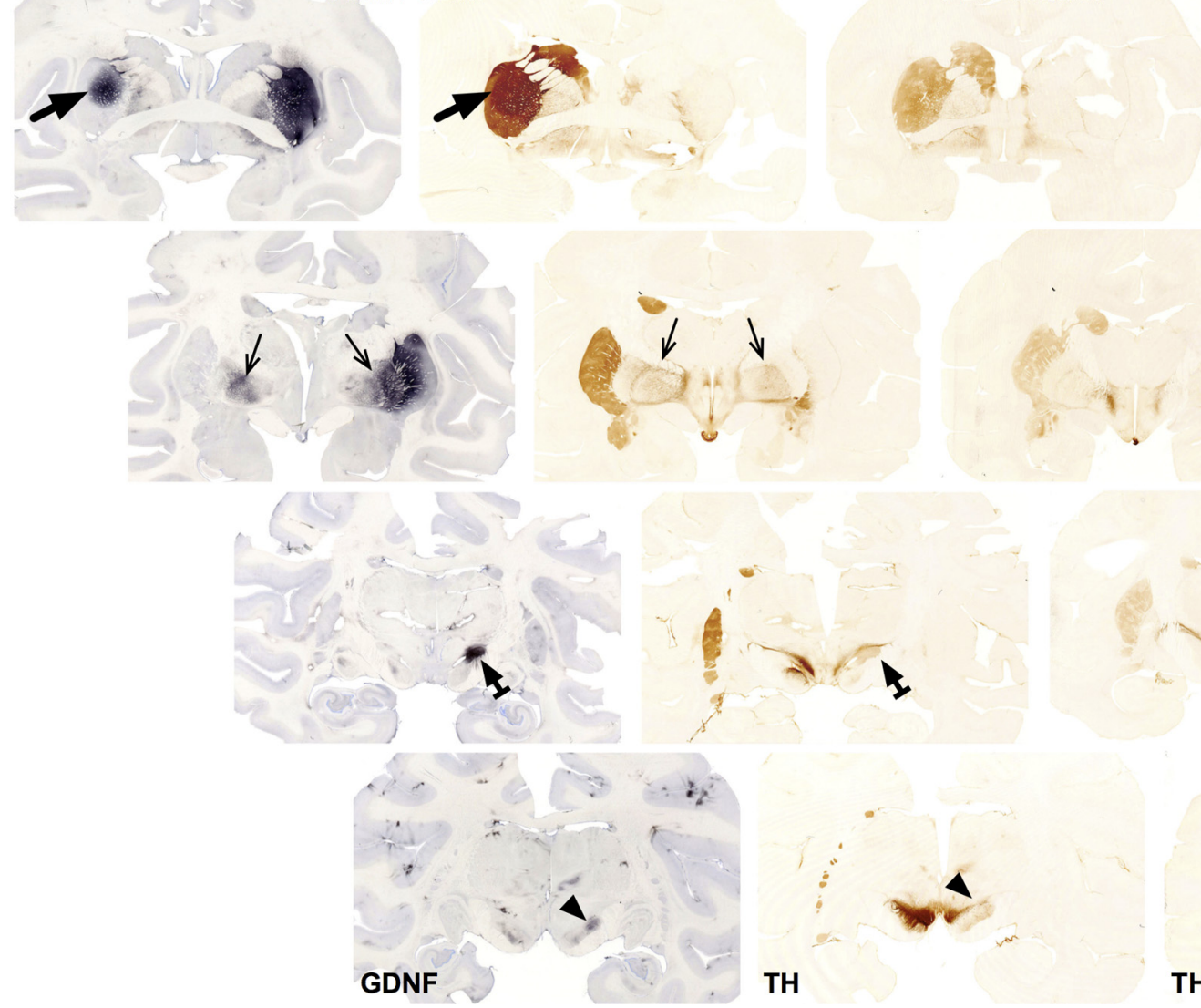

TH

Figure 3. Expression of GDNF within the basal ganglia. $\boldsymbol{A}$, Bilateral GDNF expression in the putamen at 1, 6,14 , and 24 months $(\mathrm{m})$ after AAV2-GDNF treatment. $\boldsymbol{B}$, GDNF expression was closely correlated with enhanced TH staining for adjacent sections from an AAV2-GDNF-treated macaque. TH staining in a 14-month post-treatment PBS control animal is shown for comparison. The severe MPTP lesioning of the right hemisphere is evident in the PBS controls by the substantial lack of TH staining relative to the moderately lesioned left hemisphere. Restricted GDNF expression within the left putamen of this particular animal was clearly correlated with increased TH within the same region of the putamen (thick arrows). A parallel correlation was less robust within the severely lesioned right putamen where enhanced TH was most evident in the medial and ventral aspects of the putamen. Bilaterally enhanced TH expression within the globus pallidus was evident in AAV2-GDNFtreated animals (thin arrows) and correlated with the presence of GDNF. Anterograde transportation of GDNF to the subthalamic nucleus (T'd arrows) and substantia nigra (arrowheads) was most evident in the severely lesioned right hemisphere of this animal, consistent with the greater distribution of GDNF within the right putamen. Higher magnification images of GDNF expression are available in supplemental Fig. S1, available at www.jneurosci.org as supplemental material. Scale bar, $10 \mathrm{~mm}$.

Table 2. Distribution of GDNF expression after putaminal delivery

\begin{tabular}{|c|c|c|c|c|c|c|c|c|c|c|c|c|c|}
\hline \multirow[b]{2}{*}{ NHP } & \multirow[b]{2}{*}{ Survival } & \multicolumn{6}{|c|}{ Left (moderately lesioned) } & \multicolumn{6}{|c|}{ Right (severely lesioned) } \\
\hline & & Put & $\mathrm{Cd}$ & GP & Thal & STN & SN & Put & $\mathrm{Cd}$ & GP & Thal & STN & SN \\
\hline 416 & $1 \mathrm{~m}$ & ++ & - & ++ & + & ++ & ++ & + & + & +++ & + & - & + \\
\hline 429 & $1 \mathrm{~m}$ & +++ & - & +++ & - & +++ & +++ & +++ & + & +++ & - & +++ & ++ \\
\hline $437^{a}$ & $6 \mathrm{~m}$ & +++ & + & ++ & - & +++ & ND & +++ & + & ++ & - & ++ & ND \\
\hline 438 & $6 \mathrm{~m}$ & +++ & + & +++ & - & +++ & +++ & +++ & + & +++ & - & +++ & +++ \\
\hline $415^{b}$ & $14 m$ & - & - & - & - & - & - & ++ & + & + & - & +++ & ++ \\
\hline 442 & $14 \mathrm{~m}$ & + & - & ++ & - & - & ++ & +++ & - & +++ & + & +++ & +++ \\
\hline 414 & $24 \mathrm{~m}$ & +++ & + & +++ & ++ & +++ & ++ & +++ & - & ++ & + & + & ++ \\
\hline 417 & $24 \mathrm{~m}$ & +++ & + & + & - & +++ & ++ & +++ & + & ++ & + & +++ & +++ \\
\hline
\end{tabular}

Put, Putamen; Cd, caudate nucleus; GP, globus pallidus; Thal, thalamus; STN, subthalamic nucleus; SN, substantia nigra; ND, not determined. +++ , Intense GDNF staining over $>50 \%$ of the structure; ++ , limited GDNF staining; + , some GDNF staining; -, no GDNF staining.

${ }^{a}$ Additional GDNF expression was observed in the claustrum and orbital peri-allocortex lateral and ventral to the anterior putamen in both hemispheres (supplemental Fig. S4, available at www.jneurosci.org as supplemental material). ${ }^{b}$ Left hemisphere cannula were misplaced into the cerebral cortex with no GDNF expression observed in any subcortical nuclei. GDNF expression was predominantly present in the deep cortical layers of area $8 \mathrm{~A}$. 


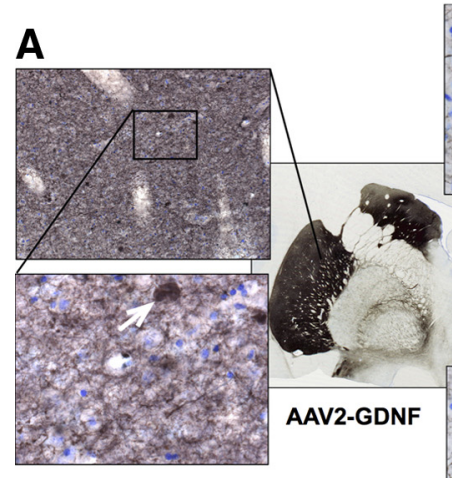

C

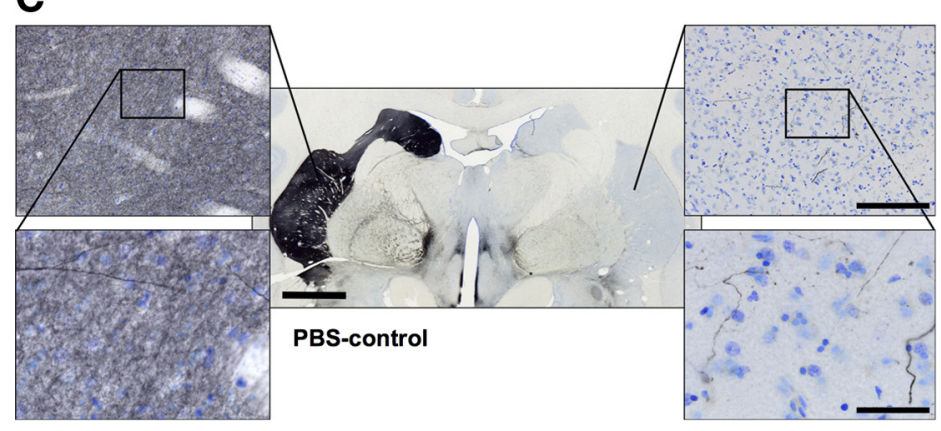

B

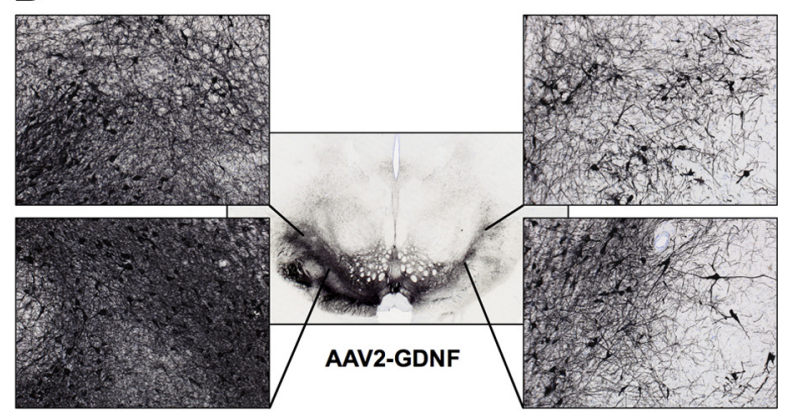

D

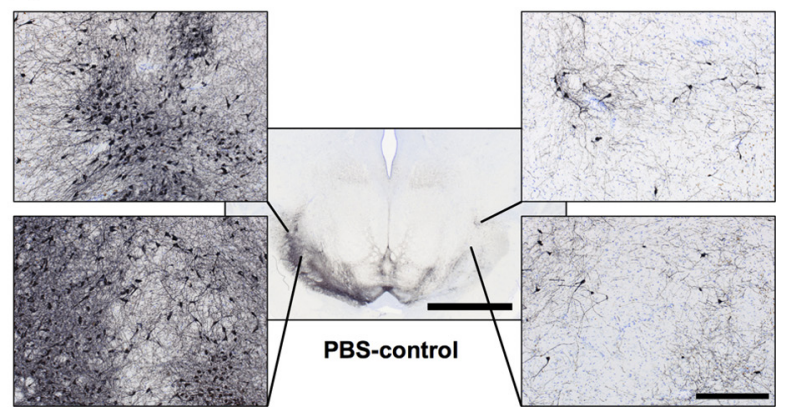

Figure 4. TH-positive fibers and neurons in the putamen and substantia nigra. $\boldsymbol{A}-\boldsymbol{D}$, AAV2-GDNF-treated macaques had more extensive TH expression within the caudate-putamen ( $\boldsymbol{A}$ ) and mid-brain $(\boldsymbol{B})$ than PBS controls $(\boldsymbol{C}, \boldsymbol{D})$. Severe MPTP-induced lesioning of the right hemisphere was evident by an almost complete lack of TH-positive fibers in the caudate-putamen; however, AAV2-GDNF treatment increased both the density and the size of TH-positive fibers. The extensive TH-positive fiber network in the left caudate-putamen prevented visualization of individual fibers but showed enhanced TH expression after AAV2-GDNF treatment and also the presence of TH-positive profiles ( $\boldsymbol{A}$, arrow) (additional images in supplemental Fig. S3, available at www.jneurosci.org as supplemental material). Bilateral upregulation of TH expression after AAV2-GDNF treatment was also evident in the substantia nigra where the asymmetry of the lesioning was evident by the number of surviving TH-positive neurons. While AAV2-GDNF-treatment increased TH expression, it was not evident whether there was an increase in the number of TH-positive neurons within the substantia nigra. Scale bars: low-magnification images, $5 \mathrm{~mm}$; medium-magnification images, $200 \mu \mathrm{m}$; high-magnification images, $50 \mu \mathrm{m}$.

was observed in the putamen of a $\mathrm{PD}$ patient that received recombinant GDNF protein infusion (Love et al., 2005).

The extent of MPTP lesioning in the mid-brain was most apparent in the right hemisphere with almost complete loss of TH-positive neurons in the substantia nigra (Fig. 4). Within the substantia nigra and ventral tegmental area (VTA), there was a considerable bilateral enhancement of TH expression in AAV2GDNF-treated macaques relative to the PBS controls and a clear increase in the density of TH-positive fibers. There was no evidence of any difference in the absolute number of TH-positive neurons between the AAV2-GDNF- and PBS-treated macaques. Unfortunately, quantification of the nigral neurons was not possible, since anatomically matched nigral sections were unavailable for all animals because of the collection of fresh tissue for GDNF and dopamine analysis. Disparity in the number of THpositive neurons was also apparent within the control animals, reflecting some inherent variability of the MPTP model within the substantia nigra. No change in $\mathrm{TH}$ immunoreactivity was evident in the left hemisphere of PBS controls when qualitatively compared with naive macaques.

\section{Correlation between GDNF distribution and TH expression}

A consistent correlation was observed in the AAV2-GDNFtreated macaques between areas of GDNF immunoreactivity and enhanced TH expression within both the moderately lesioned left putamen and the mid-brain (Fig. 3). The most evident correlation was observed in animals with a restricted region of GDNF expression, suggesting that GDNF has a strong local effect on
TH-positive fibers within the putamen. The correlation between GDNF and TH was not as strong in the severely lesioned right putamen, where the greatest increase in $\mathrm{TH}$-positive fibers was found within the ventral and medial areas of the putamen, despite broad GDNF expression throughout the putamen and globus pallidus.

\section{Safety and toxicity assessment}

A detailed safety analysis, including a full body necropsy, blood analysis, anti-AAV2 capsid and anti-GDNF antibody titers, as well as a detailed neuropathology assessment, was performed on all animals. Consistent with the 6- and 14-month endpoints ( $\mathrm{Su}$ et al., 2009), no long-term toxicity related to the AAV2-GDNF delivery into the putamen was observed during the 24-month study period. One of the AAV2-GDNF-treated animals in the 6-month group suffered a $20 \%$ decline in body weight. However, examination of the GDNF distribution in this animal showed high GDNF expression ventral to the anterior putamen on both sides that may have resulted in GDNF entering the CSF (supplemental Fig. S1, available at www.jneurosci.org as supplemental material). It appeared that the anterior cannula was positioned too ventrally in this animal, thus highlighting the need for precise positioning of the cannula to ensure accurate delivery and to prevent AAV2-GDNF leakage into the CSF or other nontargeted regions. All of the other animals maintained stable food consumption and body weights throughout the study, indicating that high GDNF expression within the basal ganglia after putaminal infusion does not influence appetite or body weight. No GDNF 
protein or antibodies against GDNF were detected in the CSF or serum. Neutralizing antibody titers against AAV capsid were measured in serum samples at up to 1:1280 postsurgery, consistent with previous reports and are now thought to be clinically unimportant (Cunningham et al., 2008; Herzog et al., 2009).

Both the AAV2-GDNF- and PBS-treated macaques exhibited enhanced GFAP expression along the cannula tracts in the putamen. Iba1-positive microglia were also found along the cannula tracts in most of the animals, including control subjects (supplemental Fig. S4, available at www.jneurosci.org as supplemental material). None of the macaques showed any evidence of an immune response beyond the immediate site of infusion, despite the extensive distribution of AAV2-GDNF and subsequent expression of GDNF in these animals.

\section{Discussion}

Extensive amelioration of functional motor deficits demonstrates the clinically meaningful restorative potential of GDNF gene therapy in both moderately and severely lesioned dopaminergic systems reminiscent of early- and late-stage PD. Although protective properties of GDNF and Neurturin have repeatedly been demonstrated (Peterson and Nutt, 2008), phase II clinical studies have failed to establish clinical efficacy (Lang et al., 2006; Ceregene press release, 2008). One possible explanation for this discrepancy between nonclinical and clinical results is the integrity of the nigrostriatal neurons at the time of treatment. Previously reported gene delivery studies in NHP have, by design, primarily investigated protection of the dopaminergic system after GDNF (Kordower et al., 2000; Eslamboli et al., 2003, 2005; Oiwa et al., 2006) or Neurturin (Li et al., 2003; Kordower et al., 2006) gene delivery in close temporal proximity to nigrostriatal lesioning. Eslamboli et al. (2005) demonstrated that GDNF expression as low as $0.04 \mathrm{ng} / \mathrm{mg}$ tissue protected nigral dopaminergic neurons from neurotoxininduced degeneration. It is possible that, although very low levels of neurotrophic factors are sufficient for protection, the much higher expression levels that we observed ( $24 \mathrm{ng} / \mathrm{mg}$ protein), combined with broad basal ganglia distribution, may be necessary for restoration of the dopaminergic neurons. With an absence of early biomarkers for idiopathic PD, it is imperative that potential therapeutics are examined in animals with extensive degeneration of the dopaminergic system, with an emphasis on providing clinically relevant restoration. Development of PD models that reliably recapitulate the progressive neurodegeneration of PD will provide valuable opportunity to investigate therapeutic effects during ongoing cellular challenge; however no such model has yet been characterized in NHP. In the current study, macaques were rendered parkinsonian by MPTP intoxication and displayed stable motor impairments for at least 3 months preceding treatment. FMT-PET before treatment confirmed the nearly complete loss of dopaminergic activity in the right putamen. This stable hemi-lesioned MPTP model was previously used in the preclinical development of AAV2-hAADC (Bankiewicz et al., 2006), with efficacy results that have proven to
B

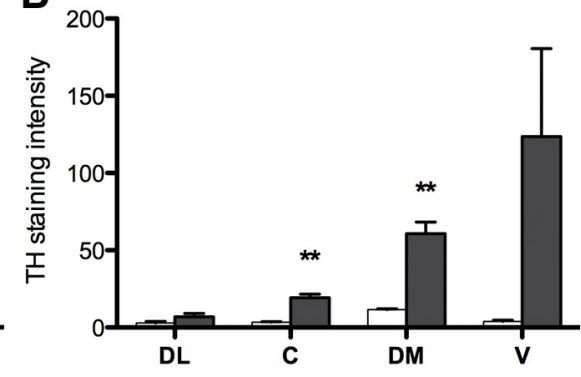

cc

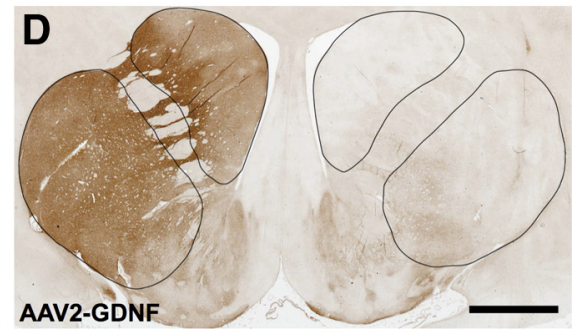

AAV2-GDNF

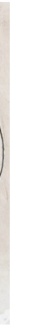

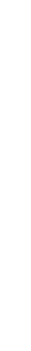

Figure 5. Restoration of dopaminergic fibers within the putamen. $A, B$, Tyrosine hydroxylase staining intensity in the moderately lesioned left $(\boldsymbol{A})$ and severely lesioned right ( $\boldsymbol{B})$ putamen of AAV2-GDNF-treated (gray bars) and PBS-treated (white bars) macaques. The mean intensity of TH staining measured in four different regions of the putamen is shown. TH staining intensity PBS- and AAV2-GDNF-treated macaques. cc, Corpus callosum; Cd, caudate nucleus; DL, dorsal lateral putamen; $C$, central putamen; Put, putamen; $v$, ventral putamen. Scale bar, $5 \mathrm{~mm}$

be highly predictive of clinical improvements observed in two independent phase I human studies (Eberling et al., 2008; Christine et al., 2009; Muramatsu et al., 2009).

Recovery of motor function in AAV2-GDNF-treated NHP coincided with significant restoration of the dopaminergic system. The bilateral increase in FMT-PET, an in-life measure of presynaptic dopaminergic activity, closely correlated with improvement in CRS scores 6 months (Eberling et al., 2009) and 24 months after AAV2-GDNF delivery. Postmortem analysis confirmed increased dopaminergic activity and showed enhanced TH-positive fiber innervation of both the moderately and the severely lesioned putamen. An absence of change in actual dopamine levels between 6,14 , and 24 months suggests that the brain rapidly accommodates the enhanced GDNF levels and that progressive improvements in functional behavior are associated with anatomical restoration of the lesioned dopaminergic system. It is important to note that additional functional recovery and FMTPET signal increases were observed beyond 6 months and that this recovery was fully maintained throughout the two-year study period. This relatively rapid but sustained recovery is supportive of the hypothesis that GDNF induces an upregulation of TH in nigrostriatal neurons that survived MPTP lesioning, and that GDNF promotes the sprouting and branching of their terminal fiber networks within the putamen. GDNF has previously been shown to induce an asymptomatic downregulation of $\mathrm{TH}$ in rats (Georgievska et al., 2002, 2004), but in concurrence with other NHP studies (Eslamboli et al., 2005), long-term GDNF expression in NHP results in a considerable enhancement of TH expression. Although we were unable to quantify neurons in the substantia nigra, no change in the number of TH-positive neurons was evident at any endpoint. In the severely lesioned hemisphere, we observed significant regrowth of TH-positive fibers, particularly in the medial and ventral aspects of the putamen, where it is likely that some residual dopaminergic fibers survive lesioning and are able to respond rapidly to GDNF expression. In 
the moderately lesioned hemisphere, we observed enhanced $\mathrm{TH}$ expression, especially within the areas of GDNF expression, enlarged $\mathrm{TH}$-positive fibers, and in the presence of large $\mathrm{TH}$ positive profiles. Although we do not know what function, if any, these TH-positive profiles have, we found no associated pathology. Such profiles were not observed in unlesioned, aged NHP that received higher doses of the same AAV2-GDNF vector (Su et al., 2009). The consistency of dopamine levels and the rate of dopamine turnover at the three different endpoints is significant from a safety perspective, as this suggests that persistent highlevel expression of GDNF in the basal ganglia after AAV2-GDNF delivery to the putamen is well tolerated.

In addition to significant efficacy data, AAV2-GDNF gene transfer in the NHP brain appeared to be well tolerated. The dose of AAV2-GDNF infused resulted in considerable spread of AAV2-GDNF into the surrounding white matter tracts and both cortical and subcortical structures. This was designed to address safety issues from both high-level GDNF expression in the putamen and other associated areas under possible circumstances where AAV2-GDNF delivery might not be completely confined to the putamen. Therefore, in addition to the putamen, AAV2GDNF-transduced neurons were observed within the globus pallidus, caudate nucleus, and cerebral cortex adjacent to the putamen or along the cannula tract. Possible adverse effects from GDNF expression were only observed in one macaque in which the cannula was too deeply positioned. All of the other macaques showed complete tolerance of the parenchymal GDNF expression. In contrast to AAV2-GDNF delivery to the mid-brain (Su et al., 2009), transport of GDNF to the substantia nigra after putaminal delivery did not induce weight loss. GDNF-induced weight loss has been reported after intraventricular protein delivery to humans (Kordower et al., 1999; Nutt et al., 2003) and also in rodents after gene delivery to the substantia nigra or hypothalamus (Tümer et al., 2006; Manfredsson et al., 2009). Overall, the lack of any apparent safety or toxicity after putaminal delivery, including immune responses, and continued responsiveness to L-dopa administration without serious adverse effect, supports initiation of a clinical AAV2-GDNF study in PD patients.

This study demonstrates that AAV2-GDNF infusion into the putamen by CED can provide broad GDNF distribution that is not limited to the putamen but includes both adjacent basal ganglia nuclei and distally located nuclei, such as subthalamic nucleus and substantia nigra. The presence of GDNF in the substantia nigra appeared to be largely dependent on achieving good distribution of GDNF in the putamen and was independent of the integrity of the dopaminergic nigrostriatal neurons, suggesting that this distribution is largely dependent on anterograde transport of AAV2-GDNF vector particles and/or GDNF protein. Therefore, it is anticipated that anterograde transport and distribution of GDNF will also occur in PD patients where the striatonigral projections to the substantia nigra pars reticulata are maintained, regardless of degree of nigrostriatal degeneration. Although it is possible that some retrograde transportation of GDNF from putamen to substantia nigra may have occurred on the moderately lesioned side, we did not observe any retrograde transport to the cortex via corticostriatal projections, which is consistent with other studies where AAV2 vectors were delivered into the putamen (Hadaczek et al., 2006; Cunningham et al., 2008). In concordance with our previous observations of AAV2GDNF transport from the substantia nigra to the striatum in aged NHP (Johnston et al., 2009) and along thalamocortical projections (Kells et al., 2009), anterograde transport is the predomi- nant model of GDNF spread within the NHP brain after AAV2GDNF delivery.

Negative results in previous neurotrophic factor phase II human clinical studies for PD investigating either recombinant GDNF protein infusion (Nutt et al., 2003; Lang et al., 2006) or AAV2-Neurturin gene transfer have been attributed to a lack of protein distribution within the basal ganglia. Inconsistencies in the delivery system have been identified as potential reasons for the disparity of results observed in the GDNF protein infusion trial (Patel and Gill, 2007). Although subjects in the open-label phase I GDNF studies reported considerable clinical benefit after CED-based delivery (Gill et al., 2003; Patel et al., 2005), the multicenter phase II study was performed with microinjection of GDNF and reported no significant clinical improvement compared with sham-treated subjects (Lang et al., 2006). In the recently reported phase II AAV2-Neurturin study (Marks and Stacy, 2009), distribution of Neurturin after microinjection into eight sites per hemisphere was calculated in two subjects to have covered only $15 \%$ of the putamen $(\sim 1.5 \mathrm{~mm}$ radius of Neurturin coverage around infusion sites), with no transport to the substantia nigra. The lack of clinical improvement in this study was attributed to an absence of Neurturin in the substantia nigra and was suggested (in the absence of supporting data) to be caused by deficient nigrostriatal retrograde transport rather than by poor coverage of the putamen. Subsequently, a second phase I/II clinical study has been initiated in which AAV2-Neurturin will be microinjected into both the putamen and substantia nigra (ClinicalTrials.gov, NCT00985517). Although we agree that neurotrophic factor distribution within both the putamen and substantia nigra is key to the achievement of the full therapeutic potential of either GDNF or Neurturin, our current findings suggest that this can be safely and reliably accomplished by improving infusion to the putamen alone. Comparison of GDNF distribution in the macaque putamen to the dimensions of the human putamen (supplemental Fig. S5, available at www.jneurosci.org as supplemental material) suggests that CED of AAV2-GDNF at the same volume ( $75 \mu \mathrm{l} /$ site) delivered in this study would cover a significant proportion of the target region, although even larger volumes are anticipated in the clinical study. To ensure optimal delivery in clinical studies, we have extensively evaluated imageguided positioning of reflux-resistant infusion cannula with realtime MR imaging of AAV2-GDNF distribution during CED (Fiandaca et al., 2008; Su et al., 2010). Only by guaranteeing that the AAV2-GDNF vector is accurately delivered into the putamen, with extensive and reproducible coverage of the targeted region, can a fair assessment be made regarding the clinical safety and efficacy of AAV2-GDNF gene therapy as a treatment for PD.

\section{References}

Bankiewicz KS, Oldfield EH, Chiueh CC, Doppman JL, Jacobowitz DM, Kopin IJ (1986) Hemiparkinsonism in monkeys after unilateral internal carotid artery infusion of 1-methyl-4-phenyl-1,2,3,6-tetrahydropyridine (MPTP). Life Sci 39:7-16.

Bankiewicz KS, Eberling JL, Kohutnicka M, Jagust W, Pivirotto P, Bringas J, Cunningham J, Budinger TF, Harvey-White J (2000) Convectionenhanced delivery of AAV vector in parkinsonian monkeys: in vivo detection of gene expression and restoration of dopaminergic function using pro-drug approach. Exp Neurol 164:2-14.

Bankiewicz KS, Sanchez-Pernaute R, Oiwa Y, Kohutnicka M, Cummins A, Eberling J (2001) Preclinical models of Parkinson's disease. Curr Protoc Neurosci Chapter 9:Unit 9.4.

Bankiewicz KS, Forsayeth J, Eberling JL, Sanchez-Pernaute R, Pivirotto P, Bringas J, Herscovitch P, Carson RE, Eckelman W, Reutter B, Cunningham J (2006) Long-term clinical improvement in MPTP-lesioned primates after gene therapy with AAV-hAADC. Mol Ther 14:564-570. 
Ceregene, Inc. (2008) Ceregene announces clinical data from phase 2 clinical trial of CERE-120 for Parkinson's disease. Press release, Nov. 26, 2008. Retrieved November 26, 2008.

Chen JJ, Swope DM (2007) Pharmacotherapy for Parkinson's disease. Pharmacotherapy 27:161S-173S.

Christine CW, Starr PA, Larson PS, Eberling JL, Jagust WJ, Hawkins RA, VanBrocklin HF, Wright JF, Bankiewicz KS, Aminoff MJ (2009) Safety and tolerability of putaminal AADC gene therapy for Parkinson disease. Neurology 73:1662-1669.

Cunningham J, Pivirotto P, Bringas J, Suzuki B, Vijay S, Sanftner L, Kitamura M, Chan C, Bankiewicz KS (2008) Biodistribution of adeno-associated virus type-2 in nonhuman primates after convection-enhanced delivery to brain. Mol Ther 16:1267-1275.

Davie CA (2008) A review of Parkinson's disease. Br Med Bull 86:109-127.

Dawbarn D, Allen SJ (2003) Neurotrophins and neurodegeneration. Neuropathol Appl Neurobiol 29:211-230.

Eberling JL, Bankiewicz KS, Jordan S, VanBrocklin HF, Jagust WJ (1997) PET studies of functional compensation in a primate model of Parkinson's disease. Neuroreport 8:2727-2733.

Eberling JL, Jagust WJ, Taylor S, Bringas J, Pivirotto P, VanBrocklin HF, Bankiewicz KS (1998) A novel MPTP primate model of Parkinson's disease: neurochemical and clinical changes. Brain Res 805:259-262.

Eberling JL, Jagust WJ, Christine CW, Starr P, Larson P, Bankiewicz KS, Aminoff MJ (2008) Results from a phase I safety trial of hAADC gene therapy for Parkinson disease. Neurology 70:1980-1983.

Eberling JL, Kells AP, Pivirotto P, Beyer J, Bringas J, Federoff HJ, Forsayeth J, Bankiewicz KS (2009) Functional effects of AAV2-GDNF on the dopaminergic nigrostriatal pathway in parkinsonian rhesus monkeys. Hum Gene Ther 20:511-518.

Eslamboli A, Cummings RM, Ridley RM, Baker HF, Muzyczka N, Burger C, Mandel RJ, Kirik D, Annett LE (2003) Recombinant adeno-associated viral vector (rAAV) delivery of GDNF provides protection against 6-OHDA lesion in the common marmoset monkey (Callithrix jacchus). Exp Neurol 184:536-548.

Eslamboli A, Georgievska B, Ridley RM, Baker HF, Muzyczka N, Burger C, Mandel RJ, Annett L, Kirik D (2005) Continuous low-level glial cell line-derived neurotrophic factor delivery using recombinant adenoassociated viral vectors provides neuroprotection and induces behavioral recovery in a primate model of Parkinson's disease. J Neurosci 25:769-777.

Fiandaca MS, Forsayeth JR, Dickinson PJ, Bankiewicz KS (2008) Imageguided convection-enhanced delivery platform in the treatment of neurological diseases. Neurotherapeutics 5:123-127.

Fiandaca MS, Varenika V, Eberling J, McKnight T, Bringas J, Pivirotto P, Beyer J, Hadaczek P, Bowers W, Park J, Federoff H, Forsayeth J, Bankiewicz KS (2009) Real-time MR imaging of adeno-associated viral vector delivery to the primate brain. Neuroimage 47 [Suppl]:T27-T35.

Forsayeth JR, Eberling JL, Sanftner LM, Zhen Z, Pivirotto P, Bringas J, Cunningham J, Bankiewicz KS (2006) A dose-ranging study of AAVhAADC therapy in Parkinsonian monkeys. Mol Ther 14:571-577.

Georgievska B, Kirik D, Björklund A (2002) Aberrant sprouting and downregulation of tyrosine hydroxylase in lesioned nigrostriatal dopamine neurons induced by long-lasting overexpression of glial cell line derived neurotrophic factor in the striatum by lentiviral gene transfer. Exp Neurol 177:461-474.

Georgievska B, Kirik D, Björklund A (2004) Overexpression of glial cell linederived neurotrophic factor using a lentiviral vector induces time- and dose-dependent downregulation of tyrosine hydroxylase in the intact nigrostriatal dopamine system. J Neurosci 24:6437-6445.

Gill SS, Patel NK, Hotton GR, O’Sullivan K, McCarter R, Bunnage M, Brooks DJ, Svendsen CN, Heywood P (2003) Direct brain infusion of glial cell line-derived neurotrophic factor in Parkinson disease. Nat Med 9:589-595.

Granholm AC, Reyland M, Albeck D, Sanders L, Gerhardt G, Hoernig G, Shen L, Westphal H, Hoffer B (2000) Glial cell line-derived neurotrophic factor is essential for postnatal survival of midbrain dopamine neurons. J Neurosci 20:3182-3190.

Hadaczek P, Kohutnicka M, Krauze MT, Bringas J, Pivirotto P, Cunningham J, Bankiewicz K (2006) Convection-enhanced delivery of adenoassociated virus type 2 (AAV2) into the striatum and transport of AAV2 within monkey brain. Hum Gene Ther 17:291-302.

Herzog CD, Brown L, Gammon D, Kruegel B, Lin R, Wilson A, Bolton A,
Printz M, Gasmi M, Bishop KM, Kordower JH, Bartus RT (2009) Expression, bioactivity, and safety 1 year after adeno-associated viral vector type 2-mediated delivery of neurturin to the monkey nigrostriatal system support cere-120 for Parkinson's disease. Neurosurgery 64:602-613.

Imbert C, Bezard E, Guitraud S, Boraud T, Gross CE (2000) Comparison of eight clinical rating scales used for the assessment of MPTP-induced parkinsonism in the Macaque monkey. J Neurosci Methods 96:71-76.

Johnston LC, Eberling J, Pivirotto P, Hadaczek P, Federoff HJ, Forsayeth J, Bankiewicz KS (2009) Clinically relevant effects of convectionenhanced delivery of AAV2-GDNF on the dopaminergic nigrostriatal pathway in aged rhesus monkeys. Hum Gene Ther 20:497-510.

Kells AP, Hadaczek P, Yin D, Bringas J, Varenika V, Forsayeth J, Bankiewicz KS (2009) Efficient gene therapy-based method for the delivery of therapeutics to primate cortex. Proc Natl Acad Sci U S A 106:2407-2411.

Kordower JH, Palfi S, Chen EY, Ma SY, Sendera T, Cochran EJ, Mufson EJ, Penn R, Goetz CG, Comella CD (1999) Clinicopathological findings following intraventricular glial-derived neurotrophic factor treatment in a patient with Parkinson's disease. Ann Neurol 46:419-424.

Kordower JH, Emborg ME, Bloch J, Ma SY, Chu Y, Leventhal L, McBride J, Chen EY, Palfi S, Roitberg BZ, Brown WD, Holden JE, Pyzalski R, Taylor MD, Carvey P, Ling Z, Trono D, Hantraye P, Déglon N, Aebischer P (2000) Neurodegeneration prevented by lentiviral vector delivery of GDNF in primate models of Parkinson's disease. Science 290:767-773.

Kordower JH, Herzog CD, Dass B, Bakay RA, Stansell J 3rd, Gasmi M, Bartus RT (2006) Delivery of neurturin by AAV2 (CERE-120)-mediated gene transfer provides structural and functional neuroprotection and neurorestoration in MPTP-treated monkeys. Ann Neurol 60:706-715.

Lang AE, Gill S, Patel NK, Lozano A, Nutt JG, Penn R, Brooks DJ, Hotton G, Moro E, Heywood P, Brodsky MA, Burchiel K, Kelly P, Dalvi A, Scott B, Stacy M, Turner D, Wooten VG, Elias WJ, Laws ER, et al (2006) Randomized controlled trial of intraputamenal glial cell line-derived neurotrophic factor infusion in Parkinson disease. Ann Neurol 59:459-466.

Li H, He Z, Su T, Ma Y, Lu S, Dai C, Sun M (2003) Protective action of recombinant neurturin on dopaminergic neurons in substantia nigra in a rhesus monkey model of Parkinson's disease. Neurol Res 25:263-267.

Lin LF, Doherty DH, Lile JD, Bektesh S, Collins F (1993) GDNF: a glial cell line-derived neurotrophic factor for midbrain dopaminergic neurons. Science 260:1130-1132.

Love S, Plaha P, Patel NK, Hotton GR, Brooks DJ, Gill SS (2005) Glial cell line-derived neurotrophic factor induces neuronal sprouting in human brain. Nat Med 11:703-704.

Manfredsson FP, Tumer N, Erdos B, Landa T, Broxson CS, Sullivan LF, Rising AC, Foust KD, Zhang Y, Muzyczka N, Gorbatyuk OS, Scarpace PJ, Mandel RJ (2009) Nigrostriatal rAAV-mediated GDNF overexpression induces robust weight loss in a rat model of age-related obesity. Mol Ther 17:980-991.

Marks WJ Jr, Stacy M (2009) Discussion of the human gene transfer protocol \#0904-981: A phase 1/2 trial assessing the safety and efficacy of bilateral intraputaminal and intranigral administration of CERE-120 (adeno-associated virus serotype 2 [AAV2]-Neurturin [NTN]) in subjects with idiopathic Parkinson's disease. Paper presented at 117th Meeting of the National Institutes of Health Recombinant DNA Advisory Committee, Bethesda, MD, June.

Matsushita T, Elliger S, Elliger C, Podsakoff G, Villarreal L, Kurtzman GJ, Iwaki Y, Colosi P (1998) Adeno-associated virus vectors can be efficiently produced without helper virus. Gene Ther 5:938-945.

Muramatsu S-i, Fujimoto K-i, Kato S, Asari S, Mizukami H, Ikeguchi K, Kawakami T, Urabe M, Kume A, Sato T, Watanabe E, Ozawa K, Nakano I (2009) Aromatic L-amino acid decarboxylase gene therapy for Parkinson's disease: results from an open-label, phase I trial. Paper presented at Twelth Annual Meeting of the American Society of Gene Therapy, San Diego, CA, May.

Nutt JG, Burchiel KJ, Comella CL, Jankovic J, Lang AE, Laws ER Jr, Lozano AM, Penn RD, Simpson RK Jr, Stacy M, Wooten GF (2003) Randomized, double-blind trial of glial cell line-derived neurotrophic factor (GDNF) in PD. Neurology 60:69-73.

Oiwa Y, Eberling JL, Nagy D, Pivirotto P, Emborg ME, Bankiewicz KS (2003) Overlesioned hemiparkinsonian non human primate model: correlation between clinical, neurochemical and histochemical changes. Front Biosci 8:a155-a166.

Oiwa Y, Nakai K, Itakura T (2006) Histological effects of intraputaminal 
infusion of glial cell line-derived neurotrophic factor in Parkinson disease model macaque monkeys. Neurol Med Chir (Tokyo) 46:267-276.

Pascual A, Hidalgo-Figueroa M, Piruat JI, Pintado CO, Gómez-Diaz R, López-Barneo J (2008) Absolute requirement of GDNF for adult catecholaminergic neuron survival. Nat Neurosci 11:755-761.

Patel NK, Gill SS (2007) GDNF delivery for Parkinson's disease. Acta Neurochir Suppl 97:135-154.

Patel NK, Bunnage M, Plaha P, Svendsen CN, Heywood P, Gill SS (2005) Intraputamenal infusion of glial cell line-derived neurotrophic factor in PD: a two-year outcome study. Ann Neurol 57:298-302.

Peterson AL, Nutt JG (2008) Treatment of Parkinson's disease with trophic factors. Neurotherapeutics 5:270-280.

Su X, Kells AP, Huang EJ, Lee HS, Hadaczek P, Beyer J, Bringas J, Pivirotto P, Penticuff J, Eberling J, Federoff HJ, Forsayeth J, Bankiewicz K (2009) Safety evaluation of AAV2-GDNF gene transfer into the dopaminergic nigrostriatal pathway in aged and Parkinsonian rhesus monkeys. Hum Gene Ther 20:1627-1640.

Su X, Kells AP, Aguilar Salegio EA, Richardson RM, Hadaczek P, Beyer J, Bringas J, Pivirotto P, Forsayeth J, Bankiewicz KS (2010) Real time MR imaging with gadoteridol predicts distribution of transgenes after convection-enhanced delivery of AAV2 vectors. Mol Ther. Advance online publication. Retrieved June 17, 2010. doi:10.1038/mt.2010.114.

Tümer N, Scarpace PJ, Dogan MD, Broxson CS, Matheny M, Yurek DM, Peden CS, Burger C, Muzyczka N, Mandel RJ (2006) Hypothalamic rAAV-mediated GDNF gene delivery ameliorates age-related obesity. Neurobiol Aging 27:459-470.

Varenika V, Kells AP, Valles F, Hadaczek P, Forsayeth J, Bankiewicz KS (2009) Controlled dissemination of AAV vectors in the primate brain. Prog Brain Res 175:163-172.

Wright JF, Qu G, Tang C, Sommer JM (2003) Recombinant adenoassociated virus: formulation challenges and strategies for a gene therapy vector. Curr Opin Drug Discov Devel 6:174-178.

Yin D, Valles FE, Fiandaca MS, Forsayeth J, Larson P, Starr P, Bankiewicz KS (2009a) Striatal volume differences between non-human and human primates. J Neurosci Methods 176:200-205.

Yin D, Valles FE, Fiandaca MS, Bringas J, Gimenez F, Berger MS, Forsayeth J, Bankiewicz KS (2009b) Optimal region of the putamen for imageguided convection-enhanced delivery of therapeutics in human and nonhuman primates. Neuroimage. Advance online publication. Retrieved September 19, 2009. doi:10.1016/j.neuroimage.2009.08.069. 